\title{
Sliding-Mode Adaptive Observer Approach to Chaotic Synchronization
}

Electrical Engineering Department, Penn State Delaware County Campus, Media, PA 19063 e-mail: azemi@psu.edu

\section{Edwin Engin Yaz}

Electrical Engineering Department, University of Arkansas, Fayetteville, AR 72701 e-mail: eyaz@uark.edu
Extensions of sliding-mode adaptive observer are presented for state reconstruction of nonlinear systems with uncertainty having unknown bounds. The observer uses nonlinear gains that are smoothened versions of classical sliding-mode gains and they are continuously updated to guarantee a globally stable observation error. This observer is applied to Chua's circuit in a chaotic synchronization scheme. A generalization to known waveform type disturbances and measurement uncertainties is pointed out. [S0022-0434(00)02304-2]

Keywords: Observer Design, Adaptive Estimation, Nonlinear Systems, Chaotic Synchronization

\section{Introduction}

In this paper, we present extensions of sliding-mode adaptive observers for state reconstruction of continuous-time nonlinear systems with uncertainties. A departure from the usual variable structure or minmax observers [1-3] is made in a way that the uncertain elements in the model are not assumed to have known bounds, but the problem is tackled via the use of estimates of these bounds which are adaptively computed. This extra degree of robustness or insensitivity is needed in the area of particular application of these observers: chaotic systems. We know that such systems are "super-sensitive" to perturbations in initial conditions and parameters [4]. Therefore, with its superb robustness properties, sliding-mode techniques have a lot of offer.

The use of variable structure techniques in state reconstruction of nonlinear systems is shown in [2] to have some advantages like allowing the presence of matched uncertain elements in the model and convergence speed over the other existing techniques like feedback linearization [5-7], extended linearization [8] and traditional Lyapunov-based techniques [9-12]. Still, this type of observer requires the knowledge of a bounding function on the uncertainty (the effect of incorrectly choosing that bound is investigated in [13]) which will not be needed in this work due to the adaptation mechanism being introduced. Other adaptive approaches in the context of variable-structure or minmax type observers can be found in [14-18].

The problem of designing a system whose behavior mimics that of another, chaotic system, is called synchronization. This is a difficult design problem since any error in the initial conditions or system parameters will result on exponential divergence of system orbits. In spite of this difficulty, the problem is of much practical significance in signal, image, speech processing and secure communications $[19,20]$. The use of nonlinear observer techniques in this area is relatively recent and the successful results are few $[21,22]$. Chua's circuit [23] will be used here in application of our sliding-mode adaptive observers.

In this work, we are going to consider systems with uncertain functions with unknown functional bounds and design a robust adaptive observer to reconstruct the state from the output measurements in such a way that the observation error will be globally stable. The observer will use continuous nonlinear gains and some generalizations of these results will be applied to models driven by known waveform type disturbances with unknown magnitude and arrival times and with measurement uncertainties. A simula-

Contributed by the Dynamic Systems and Control Division for publication in the JOURNAL OF DYNAMIC SYSTEMS, MEASUREMENT, AND CONTROL. Manuscript received by the Dynamic Systems and Control Division February 4, 2000. Associate Technical Editors: E. Misawa and V. Utkin. tion example will illustrate the design procedure and the efficacy of this technique to be used in chaotic synchronization applications.

The following notation will be used in this paper. $\mathbf{R}^{+}$is the set of non-negative real numbers. $x \in \mathbf{R}^{n}\left(A \in \mathbf{R}^{n \times m}\right)$ denotes an $n$-vector (nxm matrix) with real elements with the associated norm $\|x\|=\left(x^{T} x\right)^{1 / 2}\left(\|A\|_{s}=\bar{\lambda}^{-1 / 2}\left(A^{T} A\right)\right)$, where $(.)^{T}$ denotes transposition, $\bar{\lambda}(A)(\underline{\lambda}(A))$ denotes the maximum (minimum) eigenvalue of a symmetric matrix $A$. The symbol exp or $\mathbf{e}$ is used for the exponential function. $C^{0}$ and $C^{1}$ denotes the continuous and differentiable functions, respectively.

\section{System Model and Observer}

Consider the nonlinear, nonautonomous system model given below

$$
\dot{x}(t)=A x(t)+f(x, t)
$$

and the linear measurement equation

$$
y(t)=C x(t)
$$

where $x \in \mathbf{R}^{n}, t \in \mathbf{R}^{+}$is the system state to be reconstructed from the measurements $y \in \mathbf{R}^{p} . f(x, t)$ represents the uncertainty in system dynamics that may include parameter perturbations, external disturbances, unmodeled dynamics, etc.

We make the following assumptions about the system: (A1) $(A, C)$ is assumed to be detectable so that there exists a gain $K \in \mathbf{R}^{n \times p}$ such that $A_{0}=A-K C$ is a strictly Hurwitz matrix.

(A2) $f: \mathbf{R}^{n} \times \mathbf{R}^{+} \rightarrow \mathbf{R}^{n}$ is separable into two parts

$$
f(x, t)=f_{1}(x)+f_{2}(x, t)
$$

where the known nonlinearity $f_{1}$ satisfies a Lipshitz condition

$$
\left\|f_{1}\left(x_{1}\right)-f_{1}\left(x_{2}\right)\right\| \leqslant \alpha_{f}\left\|x_{1}-x_{2}\right\|
$$

for all $x_{1}, x_{2} \in \mathbf{R}^{n}$ where $\alpha_{f} \in \mathbf{R}^{+}$is a known constant. $f_{2}$ is, on the other hand, assumed to satisfy a classical matching condition [1]

$$
f_{2}(x, t)=P^{-1} C^{T} \zeta(y, t)
$$

where $\zeta: \mathbf{R}^{p} \times \mathbf{R}^{+} \rightarrow \mathbf{R}^{p}$ satisfies

$$
\|\zeta(y, t)\|_{s} \leqslant \sum_{i=0}^{N} c_{i} \rho_{i}(t, y)
$$

for unknown values of $c_{i} \in \mathbf{R}^{+}$, known bounded $\rho_{i} \in C^{0}$ such that $\rho_{i}: \mathbf{R}^{+} \times \mathbf{R}^{p} \rightarrow \mathbf{R}^{+}, i=0,1, \ldots, N$ and $P=P^{T}$ is the unique positive definite solution to

$$
P A_{0}+A_{0}^{T} P=-Q
$$

for some $Q=Q^{T}>0$.

(A3) The Lipshitz constant satisfies: 


$$
\alpha_{f}<\frac{1}{2} \frac{\underline{\lambda}(Q)}{\bar{\lambda}(P)}
$$

Note that we may neither know the form of $\zeta(t)$, nor the values of $c_{i}$. Still under these conditions, the adaptive observer to be proposed will estimate $c_{i}$ and at the same time reconstructs the state vector.

We will use the following nonlinear observer to reconstruct the state from the measurements $y(t)$ :

$$
\dot{\hat{x}}(t)=A \hat{x}(t)+f_{1}(\hat{x})+K(y(t)-C \hat{x}(t))+S(\hat{x}(t), y(t))
$$

where $K$ is picked to render $A_{0}$ a strictly Hurwitz matrix. The nonlinear adaptive gain

$$
S(\hat{x}(t), y(t))=\frac{P^{-1} C^{T}(y-C \hat{x}) \sum_{i=0}^{N} \hat{c}_{i}(t) \rho_{i}(t, y)}{\|y-C \hat{x}\|-\dot{h}_{1}(t) h_{2}\left(\sum_{i=0}^{N} \hat{c}_{i}(t) \rho_{i}(t, y)\right)}
$$

will be used to account for the uncertain part of dynamics. Note that with a "known" bound on the uncertain part and with, $h_{2}$ $\equiv 0$, we would get a generalized form of the classical sliding mode or minmax gain

$$
S(\hat{x}(t), y(t))=\frac{P^{-1} C^{T}(y-C \hat{x}) \sum_{i=0}^{N} c_{i}(t) \rho_{i}(t, y)}{\|y-C \hat{x}\|}
$$

for $\|y-C \hat{x}\| \neq 0$ in [1]. Therefore, we can see that the term " $-\dot{h}_{1}(t) h_{2}\left(\sum_{i=0}^{N} \hat{c}_{i}(t) \rho_{i}(t, y)\right)$ " in the denominator of $S$ in (10) functions as a (time-varying) boundary layer term (see [24], p. 290 for details). The estimates of $c_{i}$, denoted by $\hat{c}_{i}(t), i$ $=0,1, \ldots, N$ are computed as

$$
\dot{\hat{c}}_{i}(t)=q_{i}\|y-C \hat{x}\| \rho_{i}(t, y), \quad \hat{c}_{i}(0)=\hat{c}_{i 0} \in \mathbf{R}^{+}
$$

for some choice of $q_{i} \in \mathbf{R}^{+}$. Note that all $\hat{c}_{i}(t) \in \mathbf{R}^{+}$for all $t$ $\in \mathbf{R}^{+}$.

(D) The functions $h_{1}$ and $h_{2}$ satisfy the following design conditions: $h_{1} \in C^{1}, h_{1}: \mathbf{R}^{+} \rightarrow \mathbf{R}^{+}$is such that $\sup _{t \in \mathbf{R}^{+}} h_{1}(t)<\infty$ and $\sup _{t \in \mathbf{R}^{+}} \dot{h}_{1}(t)<0 . h_{2} \in C^{0}, h_{2}: \mathbf{R}^{+} \rightarrow \mathbf{R}^{+}$is any function satisfying $t h_{2}(t) \leqslant 0.5$ for all $t \in \mathbf{R}^{+}$.

One can see that functions like $\gamma_{1} e^{-\gamma_{2} t}, \operatorname{arccot}(t), \gamma_{1} /(t$ $\left.+\gamma_{2}\right)$ where $\gamma_{1}, \gamma_{2}>0$ will satisfy the properties of function $h_{1}(t)$. On the other hand, functions such as $0.5 e^{-\gamma t}$ and $0.5 /(t+\gamma)$ where $t \in \mathbf{R}^{+}, \gamma>0$ can be used for $h_{2}$. Note that there is a freedom in the choice of $h_{1}$ and $h_{2}$ which can be explored depending on the system model at hand. Moreover, due to conditions (D), the "boundary layer" term $-\dot{h}_{1}(t) h_{2}\left(\sum_{i=0}^{N} \hat{c}_{i}(t) \rho_{i}(t, y)\right) \in \mathbf{R}^{+}$. This general approach allows one to have a boundary layer that vanishes in time. If a vanishing layer is to be implemented, then $h_{1}$ should be chosen in such a way that $\dot{h}_{1}$ is decreasing slower than the convergence rate of " $C e$ ", so that no ambiguity arises in the computation of $S(\hat{x}(t), y(t))$.

\section{Convergence of the Observer}

Let us investigate the convergence property of this observer. We define the observation error $e(t) \doteq x(t)-\hat{x}(t)$ whose evolution is found from (1), (2), and (9) as

$$
\dot{e}(t)=A_{0} e(t)+f_{1}(x)-f_{1}(\hat{x})+f_{2}(x, t)-S(\hat{x}, y)
$$

and use the Lyapunov-like function candidate

$$
V\left(e, \widetilde{c}_{i}, t\right)=e^{T}(t) P e(t)+\sum_{i=0}^{N} \frac{1}{q_{i}} \widetilde{c}_{i}^{2}(t)+h_{1}(t)
$$

with $\widetilde{c}_{i}(t)=\hat{c}_{i}(t)-c_{i}, \quad i=0,1, \ldots, N$. Note that $V\left(e, \widetilde{c}_{i}, t\right)$ $\geqslant e^{T}(t) P e(t)+\sum_{i=0}^{N} 1 / q_{i} \widetilde{c}_{i}^{2}(t)>0$, so $V$ is a positive definite function. It is also true that $V$ is radially unbounded ([24], p. 64). The derivative of $V$ evaluated along the motions of $e(t)$ and $\hat{c}_{i}(t)$ is given by

$$
\begin{aligned}
\dot{V}= & 2 e^{T} P\left[A_{0} e(t)+f_{1}(x)-f_{1}(\hat{x})+f_{2}(x, t)-S\right] \\
& +2 \sum_{i=0}^{N}\left(\hat{c}_{i}(t)-c_{i}\right)\|y-C \hat{x}\| \rho_{i}(t, y)+\dot{h}_{1}(t) \\
\leqslant & -e^{T}(t) Q e(t)+2\|e(t)\|^{2} \alpha_{f} \bar{\lambda}(P)+2 e^{T}(t) P\left(f_{2}(x, t)-S\right) \\
& +2\|C e(t)\| \sum_{i=0}^{N}\left(\hat{c}_{i}(t)-c_{i}\right) \rho_{i}(t, y)+\dot{h}_{1}(t)
\end{aligned}
$$

where (4)-(7) are used together with the facts that $x^{T} P y$ $\leqslant \bar{\lambda}(P)\|x\|\|y\|$ and $y-C \hat{x}=C e(t)$.

For the nonlinear gain in (10), by using $Q \geqslant \underline{\lambda}(Q) I$ for symmetric $Q$, we obtain

$$
\begin{aligned}
\dot{V} \leqslant & -\left(\underline{\lambda}(Q)-2 \bar{\lambda}(P) \alpha_{f}\right)\|e(t)\|^{2}+2\|C e(t)\| \sum_{i=0}^{N}\left(\hat{c}_{i}(t)-c_{i}\right) \rho_{i}(t, y) \\
& +2 e^{T}(t) P\left(P^{-1} C^{T} C \zeta-\frac{P^{-1} C^{T} C e(t) \sum_{i=0}^{N} \hat{c}_{i}(t) \rho_{i}(t, y)}{\|C e(t)\|-\dot{h}_{1}(t) h_{2}\left(\sum_{i=0}^{N} \hat{c}_{i}(t) \rho_{i}(t, y)\right)}\right)+\dot{h}_{1}(t) \\
\leqslant & -\left(\underline{\lambda}(Q)-2 \bar{\lambda}(P) \alpha_{f}\right)\|e(t)\|^{2}+\frac{\|C e(t)\|-\dot{h}_{1}(t) h_{2}\left(\sum_{i=0}^{N} \hat{c}_{i}(t) \rho_{i}(t, y)\right)}{\|2\| C e(t) \|^{2} \sum_{i=0}^{N}\left(\hat{c}_{i}(t)-c_{i}\right)} \\
& \times \rho_{i}(t, y)-2\|C e(t)\| \sum_{i=0}^{N} c_{i} \rho_{i}(t, y) \dot{h}_{1}(t) h_{2}\left(\sum_{i=0}^{N} \hat{c}_{i}(t) \rho_{i}(t, y)\right)+2\|C e(t)\|^{2} \sum_{i=0}^{N}\left(\hat{c}_{i}(t)-c_{i}\right) \rho_{i}(t, y) \\
& \left.-2\|C e(t)\| \sum_{i=0}^{N}\left(\hat{c}_{i}(t)-c_{i}\right) \rho_{i}(t, y) \dot{h}_{1}(t) h_{2}\left(\sum_{i=0}^{N} \hat{c}_{i}(t) \rho_{i}(t, y)\right)\right]+\dot{h}_{1}(t)
\end{aligned}
$$




$$
\begin{aligned}
& \leqslant-\left(\underline{\lambda}(Q)-2 \bar{\lambda}(P) \alpha_{f}\right)\|e(t)\|^{2}-\frac{2\|C e(t)\| \sum_{i=0}^{N} \hat{c}_{i}(t) \rho_{i}(t, y) \dot{h}_{1}(t) h_{2}\left(\sum_{i=0}^{N} \hat{c}_{i}(t) \rho_{i}(t, y)\right)}{\|C e(t)\|-\dot{h}_{1}(t) h_{2}\left(\sum_{i=0}^{N} \hat{c}_{i}(t) \rho_{i}(t, y)\right)}+\dot{h}_{1}(t) \\
& \leqslant-\left(\underline{\lambda}(Q)-2 \bar{\lambda}(P) \alpha_{f}\right)\|e(t)\|^{2}+\left(1-2 \sum_{i=0}^{N} \hat{c}_{i}(t) \rho_{i}(t, y) h_{2}\left(\sum_{i=0}^{N} \hat{c}_{i}(t) \rho_{i}(t, y)\right)\right) \dot{h}_{1}(t) \\
& \leqslant-\left(\underline{\lambda}(Q)-2 \bar{\lambda}(P) \alpha_{f}\right)\|e(t)\|^{2}
\end{aligned}
$$

where (5), (6) and conditions $(D)$ are used. So, due to the assumption (A3), $\dot{V}$ is negative semi-definite. This means that $V(t)$ $\leqslant V(0)$ and since $h_{1}$ is uniformly bounded, for all finite initial errors, $e(t)$ and $\widetilde{c}_{i}(t)$, are uniformly bounded. Also, since $V(t)$ is lower bounded by zero and nonincreasing in time, it has a limit, $V(\infty) \doteq \lim _{t \rightarrow \infty} V(t)$. Using the results in [24], p. 107 on the properties of $V$ and $\dot{V}$, we conclude global stability of the error dynamics.

We summarize these results as follows:

Theorem 1. Let conditions $(A 1),(A 2)$, and $(A 3)$ hold, then the robust adaptive observer (9)-(11) with the design condition $(D)$ for the model (1)-(2), has globally stable observation and parameter errors.

Note that another advantage of the proposed observer is in its global stability of the error dynamics rather than the ultimate boundedness of the existing schemes [1].

\section{A Generalization}

In the following discussion, we will consider a more general system model and show how the previous observer development is applicable to this case. We start with the model

$$
\begin{gathered}
\dot{\tilde{x}}(t)=\widetilde{A} \widetilde{x}+\widetilde{f}_{1}(x)+\widetilde{f}_{2}(\widetilde{x}, t)+B_{1} d_{1}(t) \\
y(t)=\widetilde{C} \widetilde{x}(t)+g(\widetilde{x}, t)+B_{2} d_{2}(t)
\end{gathered}
$$

where $\widetilde{f}_{1}$ and $\widetilde{f}_{2}$ are the same type of nonlinearities as before. The vector function $g: \mathbf{R}^{n} \times \mathbf{R}^{+} \rightarrow \mathbf{R}^{p}$ represents the uncertainties in measurement and $d_{1}(t), d_{2}(t)$ are known waveform type (step, ramp, sinusoid, etc.) disturbances in the spirit of [25-27] having the state space models

$$
\begin{aligned}
& \dot{d}_{1}(t)=D_{1} d_{1}(t)+\sigma_{1}(t) \\
& \dot{d}_{2}(t)=D_{2} d_{2}(t)+\sigma_{2}(t)
\end{aligned}
$$

with $\sigma_{1}(t)$ and $\sigma_{2}(t)$ being "sparsely populated" impulse sequences [25]. The disturbances $d_{1}(t)$ and $d_{2}(t)$ may have unknown magnitudes and arrival times and note that it may not be possible to include these in $\widetilde{f}_{2}$ and $g$ because they may suddenly assume large magnitude changes. This more general problem formulation can be accommodated by redefining the state vector as

$$
x(t)=\left[\begin{array}{c}
\widetilde{x}(t) \\
y(t) \\
d_{1}(t) \\
d_{2}(t)
\end{array}\right]
$$

and adjoining the derivative of (17) together with (18) and (19) to (16), we obtain

$$
\begin{gathered}
\dot{x}(t)=A x(t)+f_{1}(x)+f_{2}(x, t)+\Sigma(t) \\
y(t)=C x(t)
\end{gathered}
$$

where

$$
\begin{gathered}
A=\left[\begin{array}{cccc}
\widetilde{A} & 0 & B_{1} & 0 \\
\widetilde{C} \tilde{A} & 0 & \widetilde{C} B_{1} & B_{2} D_{2} \\
0 & 0 & D_{1} & 0 \\
0 & 0 & 0 & D_{2}
\end{array}\right], \quad f_{1}(x)=\left[\begin{array}{c}
\tilde{f}_{1}(\tilde{x}) \\
\widetilde{C} \widetilde{f}_{1}(\widetilde{x}) \\
0 \\
0
\end{array}\right], \\
f_{2}(x, t)=\left[\begin{array}{c}
\tilde{f}_{2}(\tilde{x}, t) \\
\widetilde{C}_{2}\left(\widetilde{x}_{1}, t\right)+\dot{g}(\widetilde{x}, t) \\
0 \\
0
\end{array}\right] \quad \Sigma(t)=\left[\begin{array}{c}
0 \\
B_{2} \sigma_{2}(t) \\
\sigma_{1}(t) \\
\sigma_{2}(t)
\end{array}\right], \\
C=\left[\begin{array}{llll}
0 & I & 0 & 0
\end{array}\right]
\end{gathered}
$$

The assumptions of Theorem 1 need to be valid for the new set of variables and the same type of observer (of higher dimension) with the adaptive gain

$$
\begin{gathered}
\dot{\hat{x}}(t)=A \hat{x}(t)+f_{1}(\hat{x})+K(y(t)-C \hat{x}(t))+S(\hat{x}(t), y(t)) \\
S(\hat{x}(t), y(t))=\frac{P^{-1} C^{T}(y-C \hat{x}) \sum_{i=0}^{N} \hat{c}_{i}(t) \rho_{i}(t, y)}{\|y-C \hat{x}\|-\dot{h}_{1}(t) h_{2}\left(\sum_{i=0}^{N} \hat{c}_{i}(t) \rho_{i}(t, y)\right)} \\
\dot{\hat{c}}_{i}(t)=q_{i}\|y-C \hat{x}\| \rho_{i}(t, y), \quad \hat{c}_{i}(0)=\hat{c}_{i 0} \in \mathbf{R}^{+}
\end{gathered}
$$

will lead to similar convergence properties if the impulse trains $\sigma_{1}(t)$ and $\sigma_{2}(t)$ in (18) and (19) are "sufficiently sparse." That is, if these sequences are fairly infrequent, one can neglect the transients induced by $\Sigma(t)$ via making the observer (9)-(11) sufficiently fast through the choice of $K, \gamma_{1}, \gamma_{2}$, and $q_{i}$. The effect of these design parameters will be investigated by computer simulations.

Corollary 1. Let conditions (A1), (A2), and (A3) hold for the new set of variables defined in (23). Then the robust adaptive observer (9)-(11) designed based on conditions $(D)$ for the generalized model (18)-(23) has the same convergence properties given by Theorem 1 .

\section{Simulation Results}

Example: Chua's Circuit. Chua's circuit [23] is a nonlinear electronic circuit that contains four linear elements (two capacitors, one inductor, and one resistor) and a nonlinear resistor, called Chua's diode, which can be built using off-the shelf opamps (see Fig. 1). Since, Chua's circuit is endowed with an unusually rich repertoire of nonlinear dynamical phenomena, it has become a universal paradigm for chaos. Chua's circuit can be described by the following differential equations: 


$$
\begin{gathered}
C_{1} \dot{\nu}_{1}=G\left(\nu_{2}-\nu_{1}\right)-f\left(\nu_{1}(t)\right) \\
C_{2} \dot{\nu}_{2}=G\left(\nu_{1}-\nu_{2}\right)+i_{3} \\
L \dot{i}_{3}=-\left(\nu_{2}+R_{0} i_{3}\right)
\end{gathered}
$$

where $G=1 / R$ and $f\left(\nu_{1}(t)\right)=G_{b} \nu_{R}+0.5\left(G_{a}-G_{b}\right)\left(\left|\nu_{R}+E\right|\right.$ $\left.-\left|\nu_{R}-E\right|\right)$ is the $\nu$ - $i$ characteristic of the nonlinear resistor with a slope equal to $G_{a}$ in the inner region and $G_{b}$ in the outer region. For the simulation results we have used the following change of variables [23]:

$$
\begin{gathered}
x_{1}=\frac{\nu_{1}}{E}, \quad x_{2}=\frac{\nu_{2}}{E}, \quad x_{3}=\frac{i_{3}}{G E} \\
\alpha=\frac{C_{2}}{C_{1}}, \quad \beta=\frac{C_{2}}{L G^{2}}, \quad \mu=\frac{R_{0} C_{2}}{L G} \\
a=\frac{G_{a}}{G}, \quad b=\frac{G_{b}}{G}, \quad t \rightarrow \frac{t}{R C_{2}}
\end{gathered}
$$

This leads to the following model:

$$
\begin{gathered}
{\left[\begin{array}{c}
\dot{x}_{1} \\
\dot{x}_{2} \\
\dot{x}_{3}
\end{array}\right]=\left[\begin{array}{ccc}
-\alpha & \alpha & 0 \\
1 & -1 & 1 \\
0 & -\beta & -\mu
\end{array}\right]\left[\begin{array}{l}
x_{1} \\
x_{2} \\
x_{3}
\end{array}\right]-\left[\begin{array}{c}
\alpha f\left(x_{1}\right) \\
0 \\
0
\end{array}\right]} \\
y=\left[\begin{array}{lll}
1 & 0 & 0
\end{array}\right] x(t)
\end{gathered}
$$

This system can display a variety of limit sets. Figure 2 and Fig. 3 represent two different kinds of chaotic orbits, obtained from the circuit using the forthcoming parameter sets.

Using Eqs. (9)-(11), and selecting

$$
h_{1}(t)=\frac{\gamma_{1}}{t+\gamma_{2}} \quad \text { and } \quad h_{2}(t)=\frac{0.5}{t+\gamma}
$$

with

$$
\gamma_{1}=1, \quad \gamma_{2}=1, \quad \gamma=1,
$$

we obtain the following results: Figure 4 and Fig. 8 represent $x_{1}$ and its estimate for the two parameter sets. Figure 5 and Fig. 9 represent $x_{2}$ and its estimate for the two parameter sets. Figure 6 and Fig. 10 represent $x_{3}$ and its estimate for the two parameter sets. Due to extremely small errors in estimations the graphs overlay almost perfectly. Figure 7 and Fig. 11 represent the absolute error in estimation of the state variables for the two parameter sets. The following parameters have been used in these simulations:

First parameter set:

$$
\begin{gathered}
\alpha=9.100, \quad \beta=16.5811, \quad \mu=0.138083, \\
a=-1.39386, \quad b=-0.75590
\end{gathered}
$$

Second parameter set:

$$
\begin{gathered}
\alpha=10.0063, \quad \beta=16.5811, \quad \mu=0.138083, \\
a=-1.39386, \quad b=-0.75590
\end{gathered}
$$

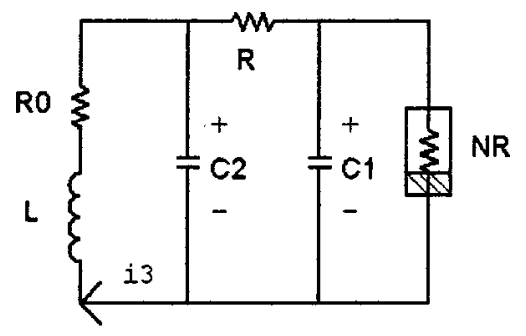

Fig. 1 Chua's circuit

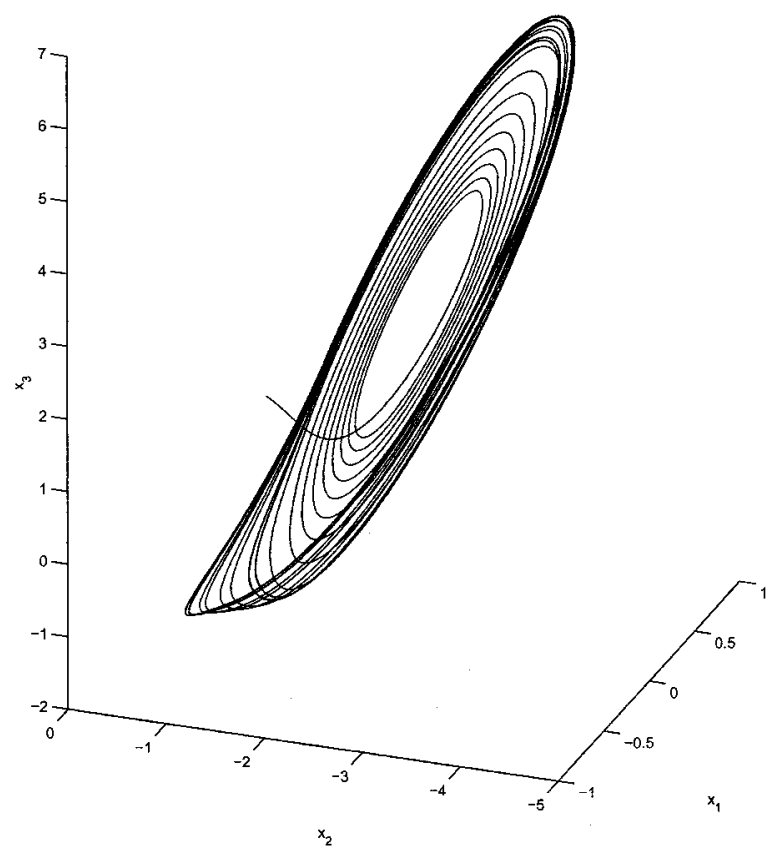

Fig. 2 Chaotic orbits for the first parameter set

Initial conditions:

$$
\begin{gathered}
x_{1}(0)=-1.0, \quad x_{2}(0)=0.1, \quad x_{3}(0)=1.0 \\
\hat{x}_{1}(0)=0.1, \quad \hat{x}_{2}(0)=-0.01, \quad \hat{x}_{3}(0)=-0.1 \\
q_{0}=0.1, \quad \hat{c}_{0}(0)=0.70
\end{gathered}
$$

The simulation results indicate that the choices of $\gamma, \gamma_{1}$, and $\gamma_{2}$ have no major effect on the performance of this observer. To show this, we included the simulation results for the second pa-

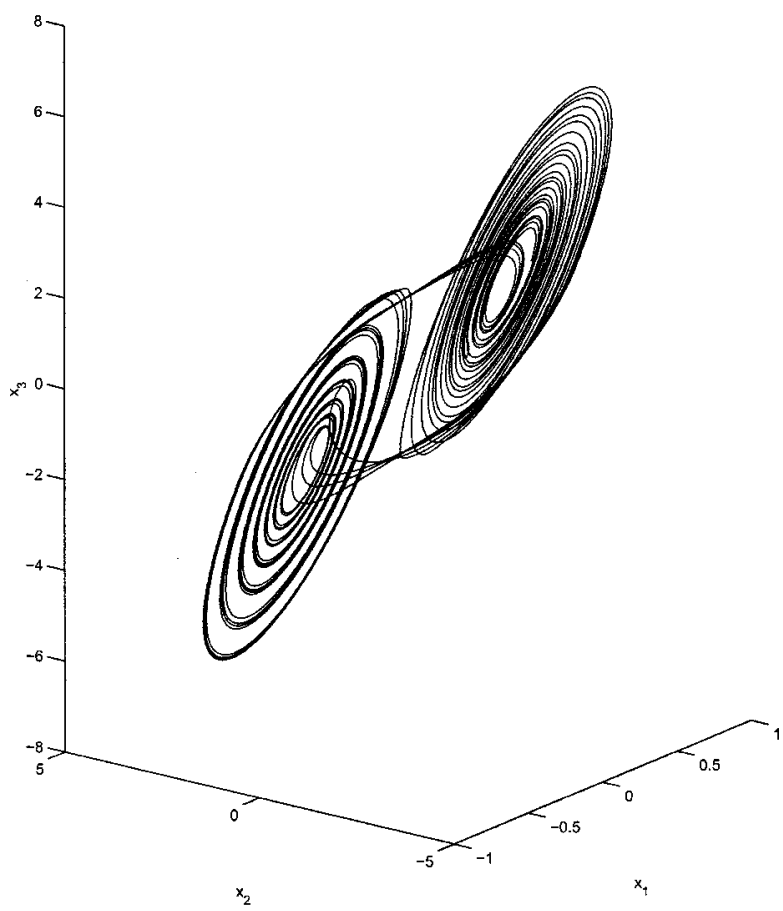

Fig. 3 Chaotic orbits for the second parameter set 


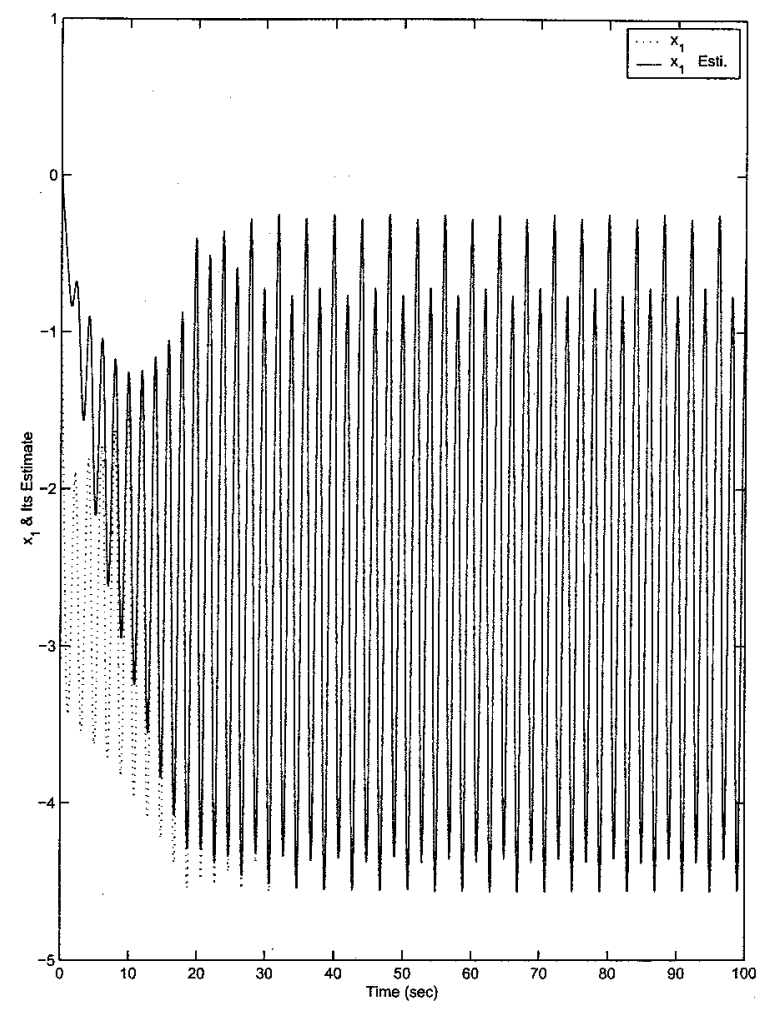

Fig. $4 x_{1}$ and its estimate for the first parameter set

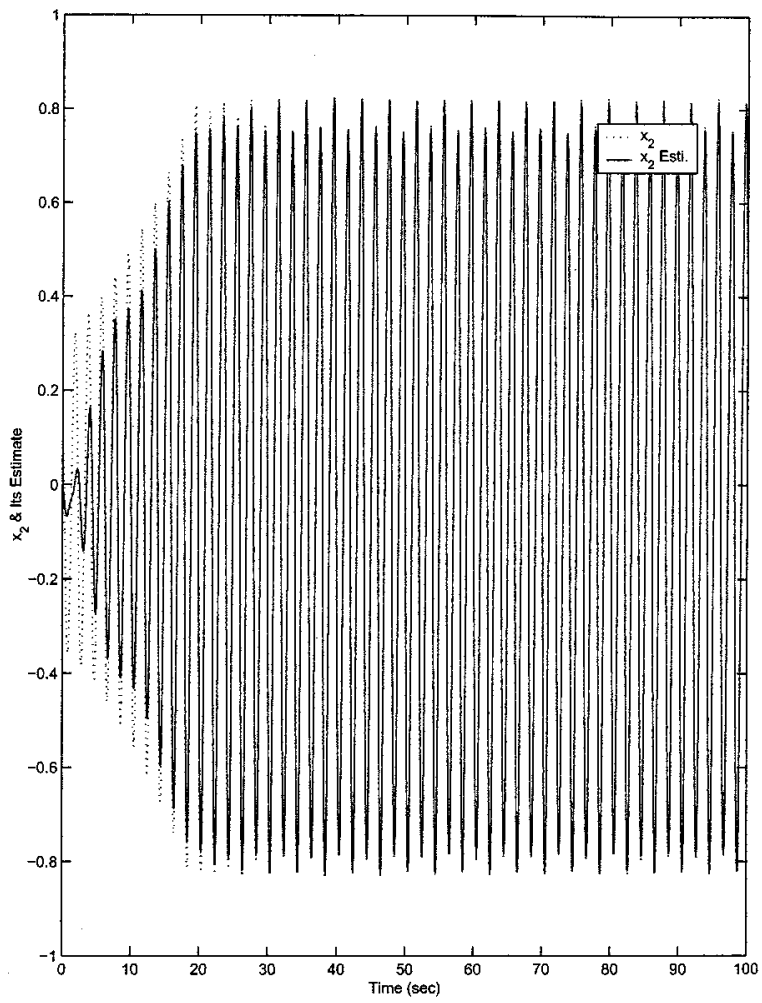

Fig. $5 \quad x_{2}$ and its estimate for the first parameter set

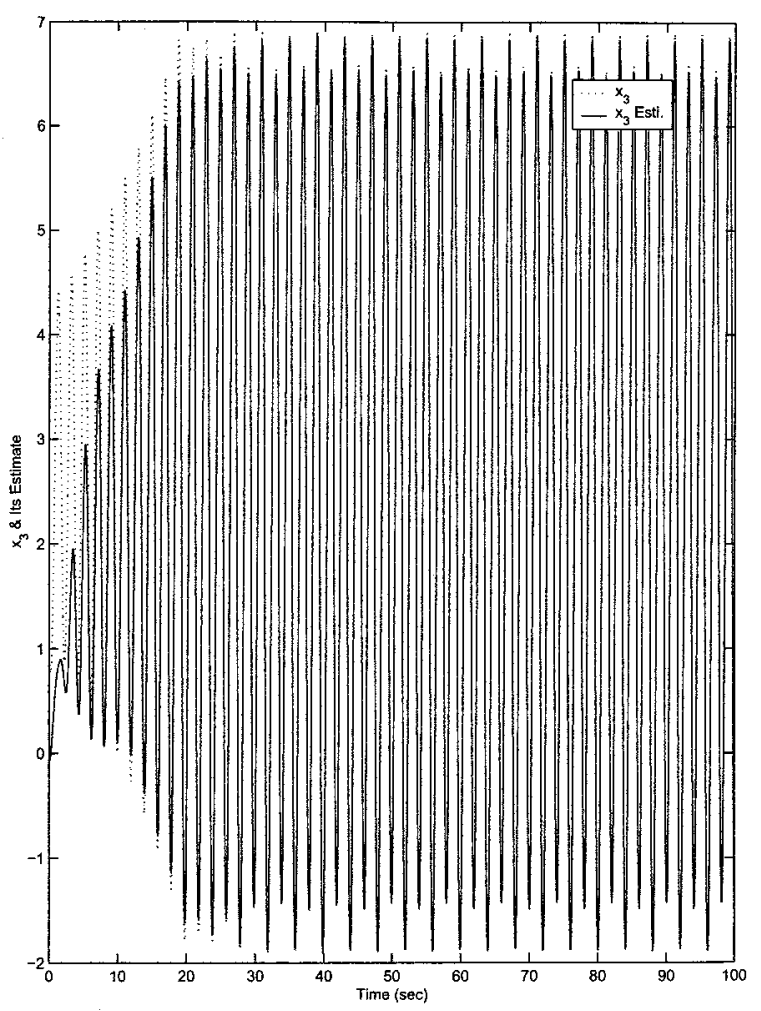

Fig. $6 \quad x_{3}$ and its estimate for the first parameter set

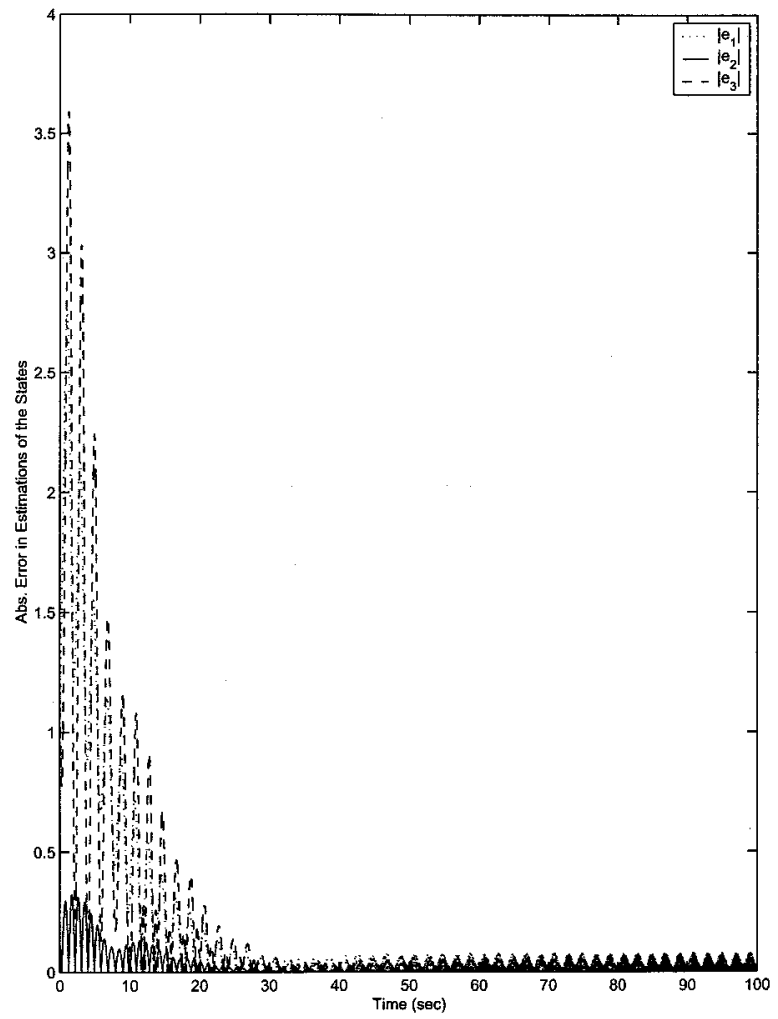

Fig. 7 Absolute error in estimation of the state variables for the first parameter set 


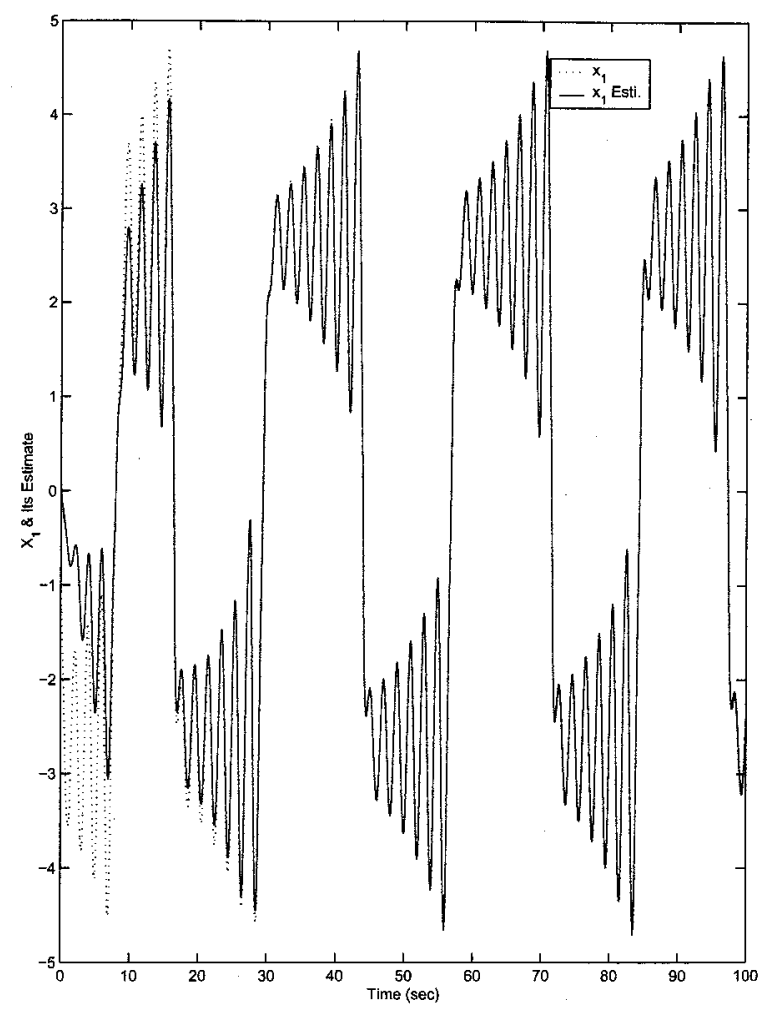

Fig. $8 \quad x_{1}$ and its estimate for the second parameter set

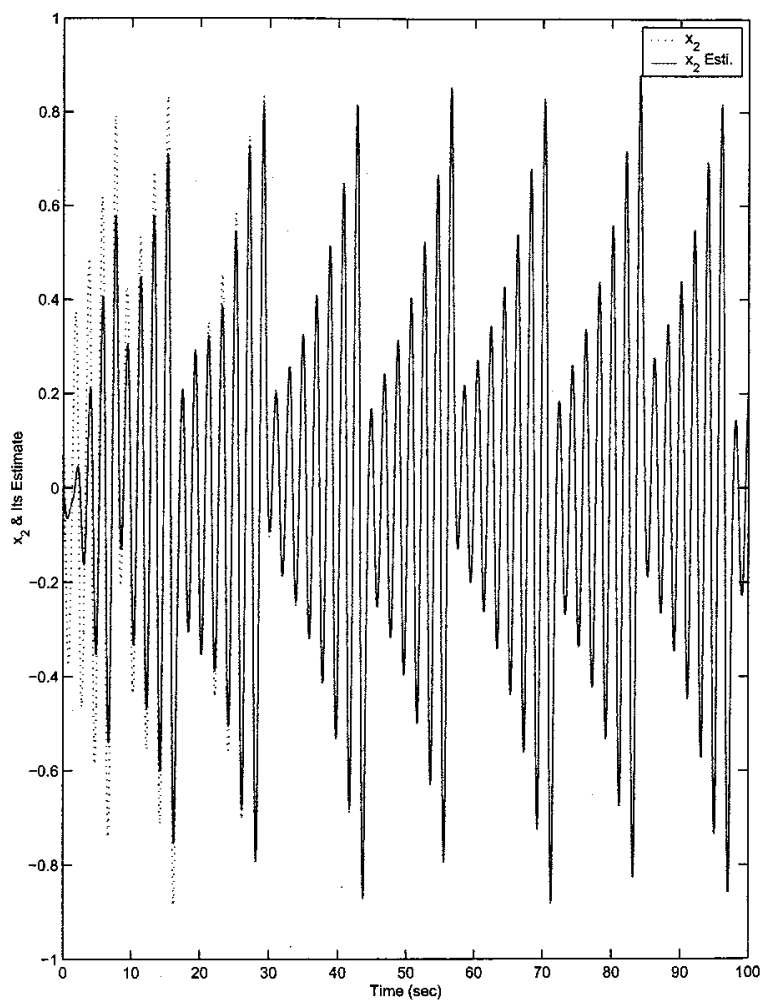

Fig. $9 x_{2}$ and its estimate for the second parameter set

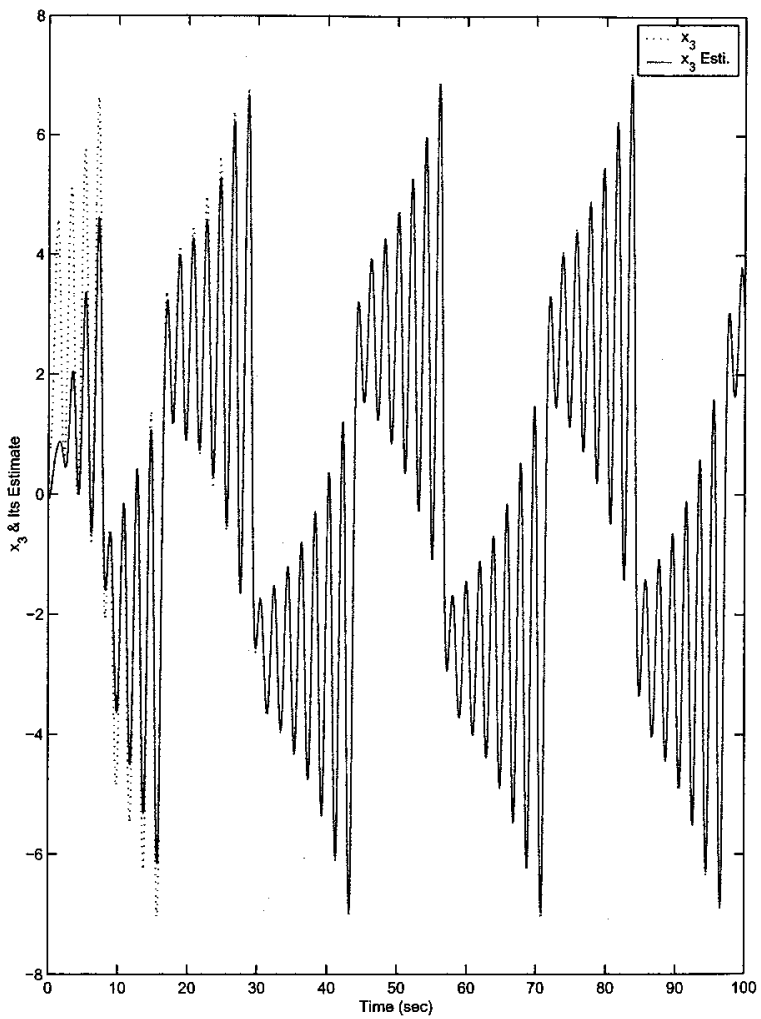

Fig. $10 x_{3}$ and its estimate for the second parameter set

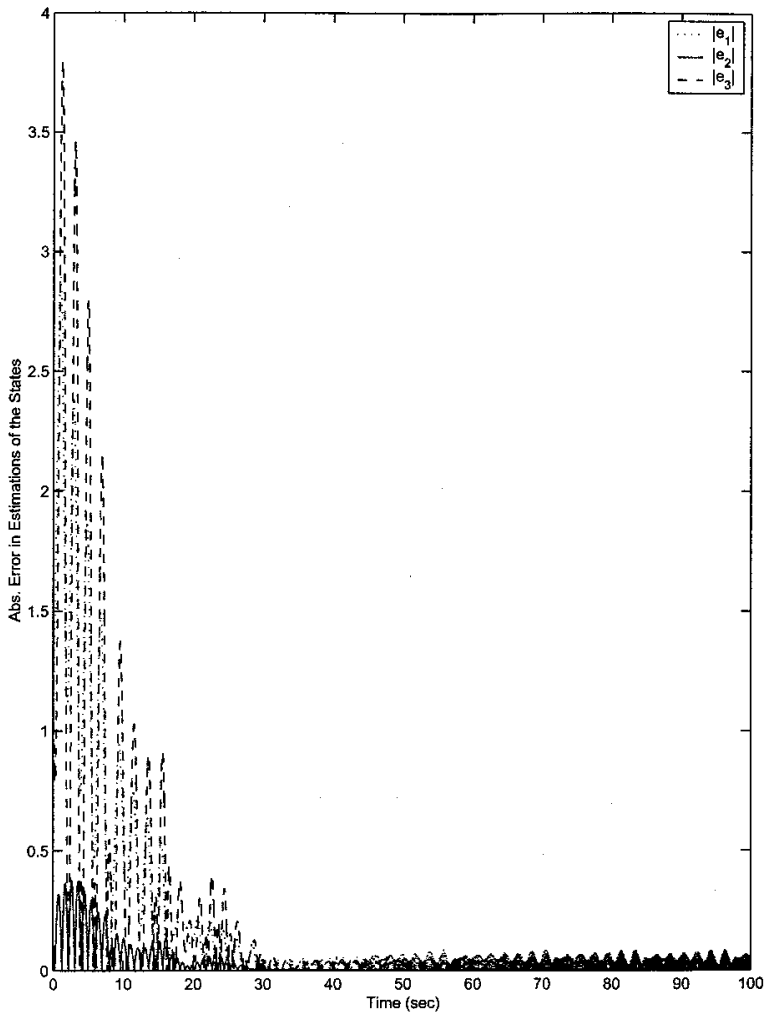

Fig. 11 Absolute error in estimation of the state variables for the second parameter set 


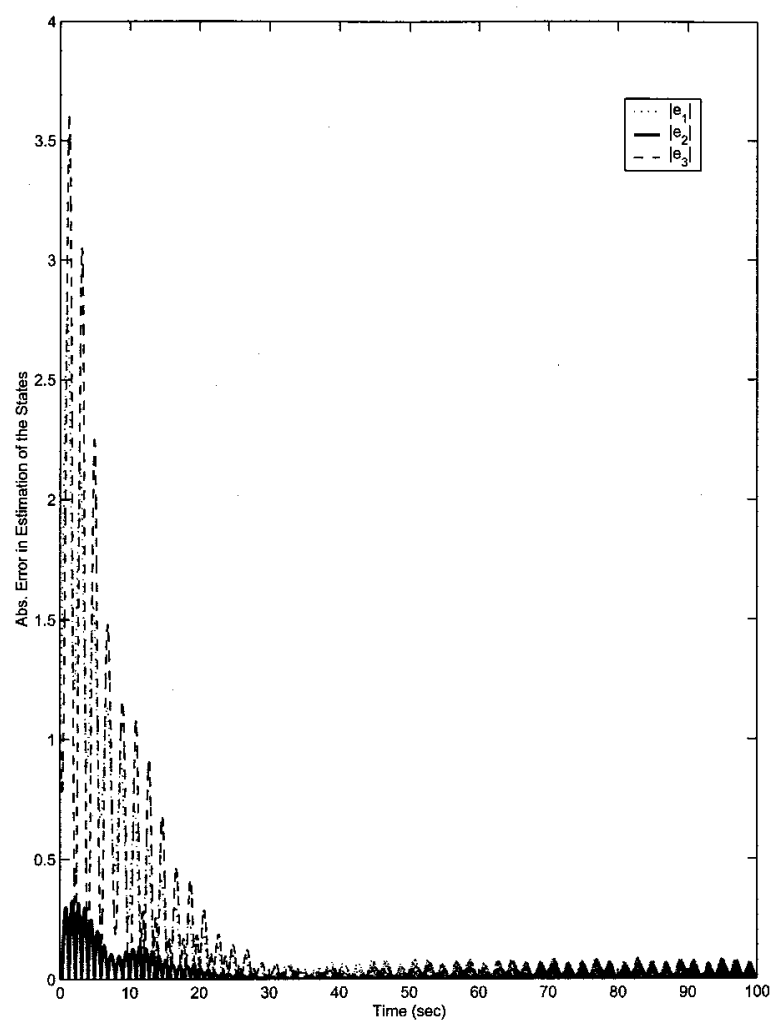

Fig. 12 Absolute error in estimation of the state variables for the second parameter set, using a different $\gamma$ value set

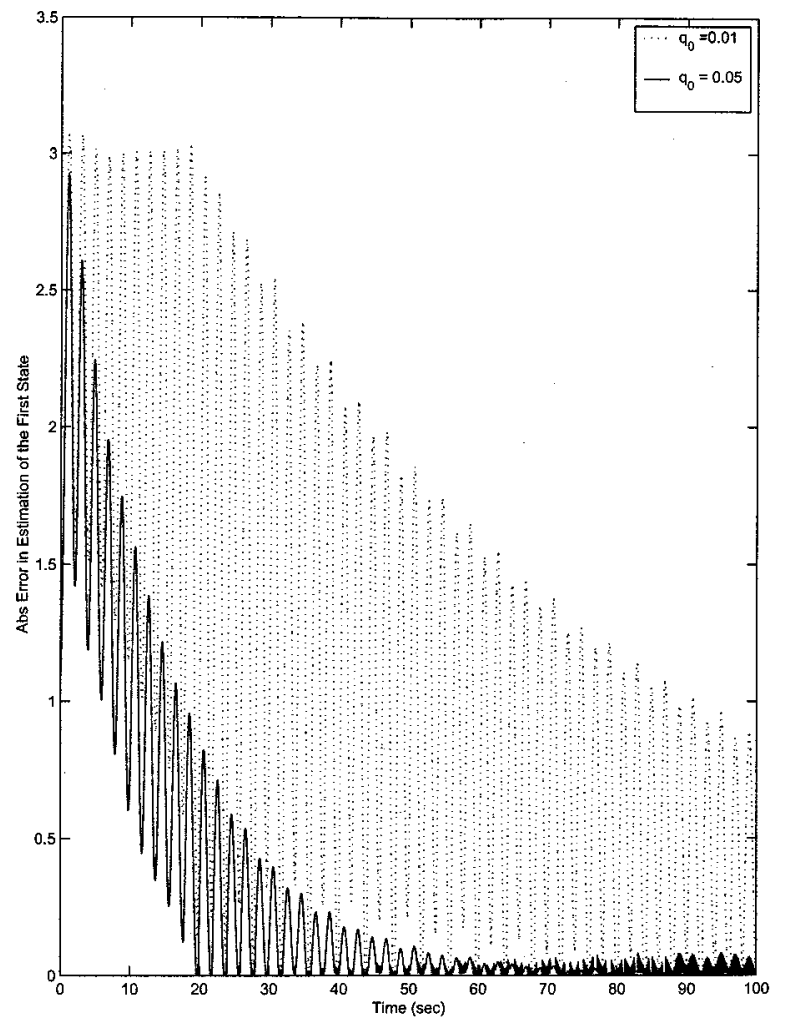

Fig. 13 Absolute error in estimation of the first state using the second parameter set, for different $q_{0}$

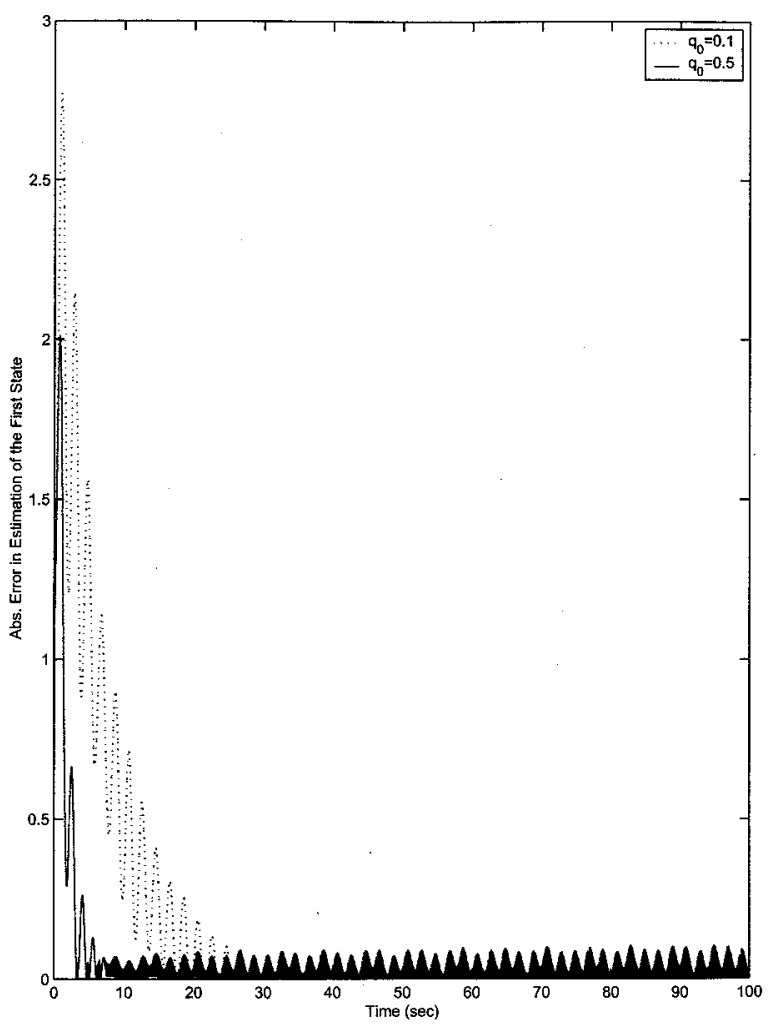

Fig. 14 Absolute error in estimation of the first state using the second parameter set, for different $q_{0}$

Table 1 MSE for the first state using second parameter set and different $q_{0}$ values

\begin{tabular}{ccc}
\hline \hline$q_{0}$ & $\begin{array}{c}\text { MSE }(0-50 \mathrm{~s}) \\
1 \text { st State }\end{array}$ & $\begin{array}{c}\text { MSE }(50-100 \mathrm{~s}) \\
\text { 1st State }\end{array}$ \\
\hline 0.01 & 3.2065 & 0.67210 \\
0.05 & 0.7921 & 0.00077 \\
0.1 & 0.3946 & 0.00080 \\
0.5 & 0.0826 & 0.00110 \\
\hline \hline
\end{tabular}

rameter set with $\gamma=10, \gamma_{1}=0.5, \gamma_{2}=5$ and illustrate in Fig. 12 the absolute estimation error which is almost an exact replica of that of Fig. 11. On the other hand, the choice of $q_{0}$ has a major impact on the performance. The higher the $q_{0}$, the faster the convergence will become. To illustrate this point, we have included plots of absolute estimation errors for the first state using the second parameter set in Fig. 13 for $q_{0}=0.01$ and $q_{0}=0.05$, and Fig. 14 for $q_{0}=0.1$ and $q_{0}=0.5$. One can see in comparison with Fig. 11, a faster and faster convergence rate is achieved with increasing $q_{0}$. Fig. 13 also shows the decrease in the steady-state error. This decrease continues to a certain point for $q_{0}$, after which it starts to increase. To further illustrate this effect, in Table 1, we have calculated the mean squared error (MSE) for the parameters used in producing Fig. 13 and Fig. 14, for two different time intervals.

\section{Conclusion}

Nonlinear state observers are presented which adaptively reconstruct the state of an uncertain system from output measurements. These observers use a modified version of nonlinear sliding mode gains in a particularly smoothed form with the resulting theoreti- 
cal simplicity and implementation ease and do not require the knowledge of bounds on the uncertain functions. A generalization to problems with measurement uncertainties and known waveform type disturbances has been shown to be possible. The example taken from chaotic synchronization, illustrates the use of the proposed observer and its success in correctly estimating the state in simulations.

\section{References}

[1] Walcott, B. L., and Zak, S. H., 1987, "State observation of nonlinear uncertain dynamical systems," IEEE Trans. Autom. Control, 32, pp. 166-170.

[2] Walcott, B. L., Corless, M. J., and Zak, S. H., 1987, "Comparative study of non-linear state-observation technique," Int. J. Control, 45, pp. 2109-2132.

[3] Slotine, J-J. E., Hedrick, J. K., and Misawa, E. A., 1986, 'On sliding observers,'” ASME J. Dyn. Syst., Meas., Control, 109, pp. 245-252.

[4] Ruelle, D., 1989, Elements of Differentiable Dynamics and Bifurcation Theory, Academic Press, San Diego.

[5] Bestle, D., and Zeitz, M., 1983, "Canonical form observer design for nonlinear time-variable systems,', Int. J. Control, 38, pp. 419-431.

[6] Krener, A. J., and Respondek, W., 1985, "Nonlinear observer with linearizable error dynamics," SIAM J. Control Optim., 23, pp. 197-216.

[7] Isidori, A., 1985, Nonlinear Control Systems: An Introduction, SpringerVerlag, Berlin.

[8] Baumann, W., and Rugh, W., 1986, "Feedback control of non-linear systems by extended linearization,", IEEE Trans. Autom. Control, 31, pp. 40-47.

[9] Thau, F. E., 1973, "Observing the state of non-linear dynamic systems,' Int. J. Control, 18, pp. 471-479.

[10] Kou, S. R., Elliot, D. L., and Tarn, T. J., 1975, "Exponential observer for nonlinear dynamic systems,'” Inf. Control., 29, pp. 204-216.

[11] Vidyasagar, M., 1980, "On the stabilization of nonlinear systems using state detection," IEEE Trans. Autom. Control, 25, pp. 504-509.

[12] Yaz, E., and Azemi, A., 1993, "Observer design for discrete and continuous nonlinear stochastic systems,'” Int. J. Syst. Sci., 21, pp. 2289-2302.

[13] Yaz, E., and Azemi, A., 1993, "Sliding mode observers for nonlinear models with unbounded noise and measurement uncertainties,' Dynamics \& Control, 3, pp. 217-235.
[14] Corless, M., and Leitmann, G., 1983, “'Adaptive control of systems containing uncertain functions and unknown functions with uncertain bounds," JOTA, 41, pp. $155-168$.

[15] Chen, Y. H., 1989, “Modified adaptive robust control system design,' Int. J. Control, 49, pp. 1869-1882.

[16] Chen, Y. H., 1989, "State estimation for non-linear uncertain systems: a design based on properties related to the uncertainty bound,' Int. J. Control, 52, pp. 1131-1146.

[17] Chen, Y. H., 1992, “Adaptive robust control of uncertain systems with measurement noise," Automatica, 28, pp. 715-728.

[18] Yoo, D. S., and Chung, M. J., 1992, “A variable structure control with simple adaptation laws for upper bounds on the norm of the uncertainties,' IEEE Trans. Autom. Control, 37, pp. 860-865.

[19] Carroll, T. L., and Pecora, L. M., 1991, "Synchronizing chaotic circuits," IEEE Trans. Circuits Syst., 38, pp. 453-456.

[20] Ogorzalek, M. J., 1993, "Taming Chaos-Part I: Synchronization," IEEE Trans. Circuits Syst., I: Fundam. Theory Appl., 40, pp. 693-699.

[21] Nijmeijer, H., and Mareels, I. M. Y., 1997, “An observer looks at synchronization,' IEEE Trans. Circuits Syst., I: Fundam. Theory Appl., 44, pp. 882 890.

[22] Sobiski, D. J., and Thorp, J. S., 1998, “PDMA-1: Chaotic Communication via the EKF,', IEEE Trans. Circuits Syst., I: Fundam. Theory Appl., 45, pp. 194197.

[23] Chua, L., Wu, C., Hung, A., and Zhong, G., 1993, “A universal circuit for studying and generating chaos-part I: routes to chaos,' IEEE Trans. Circuits Syst., I: Fundam. Theory Appl., 40, pp. 732-744.

[24] Slotine, J-J. E., and Li, W., 1991, Applied Nonlinear Control, Prentice-Hall, Englewood Cliffs, NJ.

[25] Johnson, C. D., 1980, "Disturbance accommodation control: an overview of the subject,' J. Interdis. Model and Simul., 3, pp. 1-29.

[26] Yaz, E., and Azemi, A., 1993, "Variable structure observer with a boundarylayer for correlated noise/disturbance models and disturbance minimization,', Int. J. Control, 57, pp. 1191-1206.

[27] Azemi, A., and Yaz, E., 1993, 'Dynamic disturbance minimization control for discrete nonlinear stochastic systems,'” Optimal Control Applications \& Methods, 14, pp. 181-194. 